\title{
Le Couchsurfing : une forme d'hospitalité touristique entre familiarité et altérité
}

Couchsurfing: a type of touristic hospitality in-between familiarity and alterity

Pauline Neveu

\section{OpenEdition}

Journals

Édition électronique

URL : http://journals.openedition.org/tourisme/1394

DOI : $10.4000 /$ tourisme. 1394

ISSN : 2492-7503

Éditeur

Éditions touristiques européennes

\section{Référence électronique}

Pauline Neveu, «Le Couchsurfing : une forme d'hospitalité touristique entre familiarité et altérité ", Mondes du Tourisme [En ligne], 13|2017, mis en ligne le 30 décembre 2017, consulté le 20 mars 2020. URL : http://journals.openedition.org/tourisme/1394; DOI : https://doi.org/10.4000/tourisme.1394

Ce document a été généré automatiquement le 20 mars 2020.

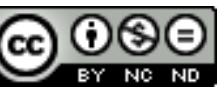

Mondes du tourisme est mis à disposition selon les termes de la licence Creative Commons Attribution - Pas d'Utilisation Commerciale - Pas de Modification 4.0 International. 


\title{
Le Couchsurfing : une forme d'hospitalité touristique entre familiarité et altérité
}

\author{
Couchsurfing: a type of touristic hospitality in-between familiarity and alterity
}

\author{
Pauline Neveu
}

Le tourisme n'a pas toujours une réputation favorable: on le caractérise tantôt de factice, parfois de destructeur, et personne ne souhaite être perçu comme un touriste (Urbain, 1991). Néanmoins, ce qu'on lui reproche sûrement le plus souvent, c'est son manque de rapport social avec l'altérité (Godbout, 1997). Et pourtant, le secteur touristique voit émerger des formules de voyage innovantes, qui suscitent des rencontres entre visiteurs et résidents. C'est le cas du réseau d'hospitalité Couchsurfing. Cette plateforme permet l'hébergement gratuit entre membres. Le réseau suit le fonctionnement suivant : un touriste - aussi appelé surfeur, puisqu'il "surfe » les canapés ${ }^{1}$ - désirant trouver un hébergeur sélectionne le profil d'un autre couchsurfeur dans la base de données qu'offre le réseau. Il lui envoie ensuite une "demande de canapé " afin de savoir si le résident souhaite l'accueillir. Si l'hébergeur accepte la requête, les deux personnes cohabiteront pendant deux à trois jours en moyenne (Bialski, 2009). Les adeptes de cette forme de voyage sont majoritairement des jeunes, la moyenne d'âge étant de 28 ans selon les chiffres du réseau ${ }^{2}$, ils proviennent des pays occidentaux et sont issus des classes moyennes, voire moyennes-supérieures de leur société. 
Figure 1. Statistiques sur l'âge et le genre des couchsurfeurs

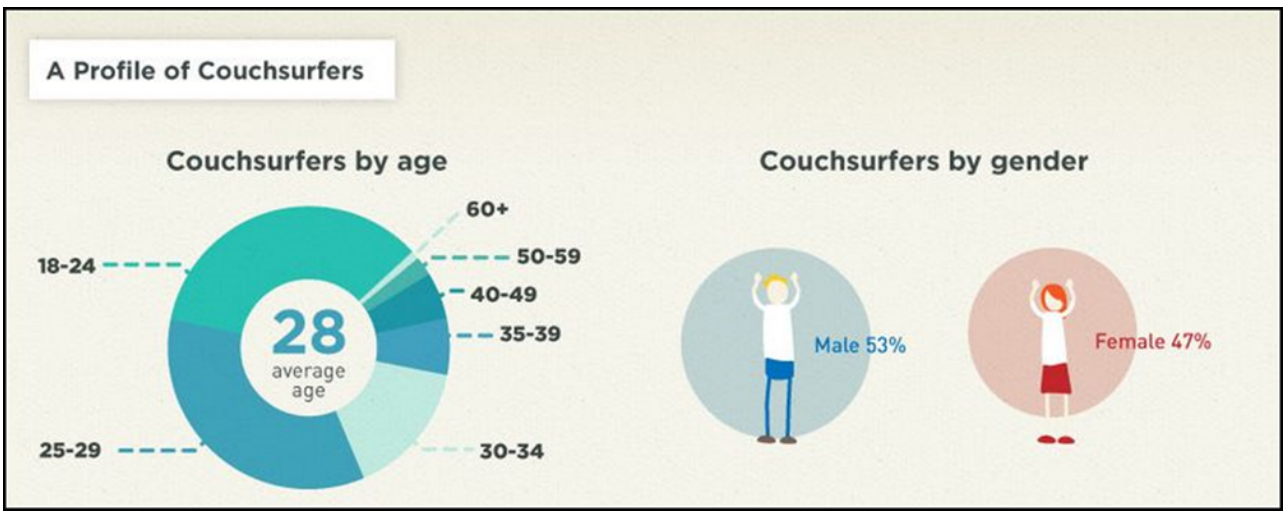

Source : http://www.couchsurfing.org/statistics.html (consulté en juin 2014)

2 Si l'hospitalité ainsi que l'accueil de voyageurs ne sont pas des activités nouvelles, leur médiation et systématisation via un réseau en ligne implique une évolution de l'expérience touristique des membres. En particulier, comme les couchsurfeurs qui se rencontrent sont des inconnus les uns pour les autres, cela modifie les conceptions des voyageurs en mettant l'emphase touristique sur la personne rencontrée plus que sur la ville visitée. La réflexion qui suit débute par une présentation des intérêts et limites de la méthodologie qualitative qui a été employée dans cette recherche. Puis, nous montrerons en quoi l'usage des nouvelles technologies dans le processus de rencontre entre les membres reproduit des logiques de sélection sociale classiques: contrairement à ce que l'idéologie du réseau met en avant, un entre-soi social est constitué. Cet entre-soi, initié en ligne, permet une forme de résolution pratique de la tension touristique entre familiarité et altérité. Dans leurs discours, les couchsurfeurs alternent entre le plaisir et la "surprise» touristique de rencontrer une nouvelle personne et, dans le même temps, le confort interactionnel relatif qu'interagir avec un couchsurfeur implique. Ces données sont riches d'enseignement puisqu'elles illustrent une forme d'équilibre touristique ${ }^{3}$ entre l'habituel et le différent au sein même d'une relation hospitalière entre inconnus - situation qui, jusqu'à présent, a peu été étudiée. Enfin, la troisième section s'attache à présenter les implications touristiques du contexte Couchsurfing. Car, si les surfeurs se perçoivent comme vivant une expérience alternative, ils ne visitent pas la ville si différemment des autres voyageurs. De façon quelque peu paradoxale par rapport à leurs discours, les couchsurfeurs ne vont pas manquer de faire les attractions les plus classiques lors de leur séjour ; cela s'explique par l'importance que revêt la rencontre avec un inconnu dans leur conception du voyage. Ainsi, Couchsurfing est une formule hybride, qui répond à des aspirations touristiques, contenues dans une situation hospitalière, à mi-chemin entre familiarité communautaire et altérité individuelle.

\section{Une méthodologie de l'accueil}

Deux méthodes principales, mises en place à Paris, ont servi à recueillir les données nourrissant l'analyse qui suit. Dans un premier temps, une observation participante à « l'intérieur » de la rencontre entre couchsurfeurs a été mise en place. J'ai accueilli sept touristes pendant trois jours en moyenne. J'ai ainsi vécu avec ces personnes et partagé avec elles des activités, telles que des repas ou encore des visites touristiques. Cet accès 
privilégié a permis de rédiger des descriptions des actions et discours des couchsurfeurs en visite dans la capitale française. À cette ethnographie s'ajoutent des entretiens semidirectifs menés chez moi avec ces voyageurs à la fin de leur séjour. De plus, des entretiens ont également été menés avec neuf hébergeurs, qui ont répondu à un appel laissé sur le forum Couchsurfing des accueillants parisiens. Ces seize entrevues ont été entièrement retranscrites grâce au logiciel Sonal, afin de mener une analyse thématique qui a permis de transposer le corpus en différents thèmes représentatifs du contenu, thèmes qui ont été constitués en fonction de nos questions de recherche (Paill é et al., 2008). Finalement, deux à trois photographies de leur séjour ont été collectées auprès des surfeurs, comme méthodologie complémentaire. Il leur a été demandé de sélectionner des clichés qui « représentaient le plus leur expérience parisienne » et une analyse visuelle des images reçues a été mise en place.

\section{Lorsque la participation est intrinsèque à l'observation ethnographique}

4 Une des pierres angulaires du réseau repose sur la volonté de ses membres à rencontrer un inconnu afin d'interagir avec ce dernier. L'entrée sur le terrain pour une sociologue peut donc se voir, dans un premier temps, simplifiée : nul besoin de se présenter dès le départ comme faisant une recherche afin d'accéder au lieu de l'étude, ou encore d'expliquer sa présence. De la même façon, poser des questions personnelles n'est pas perçu comme envahissant ou inquisiteur : même si les surfeurs savent qu'une étude sur Couchsurfing est menée, cela leur parait normal que leur hôtesse cherche à les connaître. La discussion est, en effet, intrinsèque aux échanges entre couchsurfeurs, il s'agit de découvrir l'autre, de faciliter l'interaction sociale, ou encore d'obtenir des informations à propos des endroits à visiter et du lieu de vie de l'hébergeur. Le partage de moments et d'activités entre surfeur et hôte est primordial, sous peine de ne pas répondre à l'impératif de rencontre et d'échange du réseau. C'est pourquoi mes invités me demandaient de passer du temps avec eux, tout comme je le souhaitais moi-même. Dans un tel contexte, adopter uniquement des attitudes distanciées d'observatrice, dans le but de répondre à des impératifs de "neutralisation scientifique ", n'est ni possible ni souhaitable. Il en va de la survie du terrain : tout enquêteur qui hébergerait en ne s'impliquant pas suffisamment dans l'interaction n'obtiendrait que des commentaires négatifs et des échanges peu fructueux (Soulet et al., 2010). Nous voyons donc apparaître l'un des revers de ce type de méthodologie proche d'une situation de laboratoire social : elle ne permet pas une posture de chercheur fortement désengagée ; bien au contraire, l'entrée spécifique sur le terrain plonge l'observateur dans l'interaction sans possibilité d'en sortir tant que l'échange a lieu. Les attitudes de l'enquêteur s'en trouvent rigidifiées, mais cela n'est que temporaire, et il semble cohérent dans ce genre de méthodologies d'interrompre momentanément certaines postures scientifiques afin de permettre une vraie «perméabilité communicationnelle » avec les enquêtés (Favret-Saada, 1990). Il a ainsi été beaucoup plus facile de reprendre les données accumulées sur le terrain une fois leur collecte terminée. De cette façon, la retranscription des entretiens, la relecture des observations ethnographiques ainsi que l'analyse des photographies des voyageurs ont été très riches d'enseignement une fois hors du terrain. Cette segmentation temporelle de la recherche a permis d'apaiser le paradoxe ethnographique qui veut que le chercheur observe des normes et des pratiques dans le même temps qu'il doit se 
conformer à ces dernières. Cela est particulièrement important dans une étude sur l'hospitalité, dont le terrain est coloré de rapports de pouvoir.

\section{Une enquête imbriquée dans les rapports de pouvoir de l'hospitalité}

5 L'hospitalité est un phénomène ambivalent: elle est tantôt recherchée et suscitée, tantôt fuite et diabolisée. Comme le formule très bien A. Montandou, (2001, p. 15) : « N'être qu'un hôte est donc à la fois un don magnifique et une situation inconfortable, précaire, soumise aux lois du temps de l'accueil. Hospitalité désirée et hospitalité douloureuse. Éphémère et précaire, conditionnée par des lois, celle du séjour sous condition." C'est pourquoi la cohabitation et tout ce qu'elle implique - discussions, partage de l'espace, repas, entre autres - peut se révéler aussi sympathique que désagréable pour les deux parties impliquées. Cette ambivalence se retrouve jusque dans l'analyse du terme « hôte ». En effet, hospes, l'hôte, est étymologiquement proche de hotis, l'ennemi, l'étranger (Durfourmantelle et al., 1997 ; Grassi, 2001, p. 28). Les couchsurfeurs connaissent bien cette dimension menaçante de l'hospitalité et des rapports de pouvoir qui la régissent, particulièrement les surfeurs, qui sont les plus fragiles dans la relation entre maître de maison et invité. Ces derniers risquent en effet de perdre leur toit, de l'argent ainsi que l'un de leur seul ancrage social dans le lieu visité s'ils ne respectent pas les conditions d'accueil de l'hébergeur. De ce fait, le type de méthodologie employé dans cette recherche aboutit à des données colorées par l'asymétrie entre accueillants et accueillis, ces derniers étant bien conscients de leur position de faiblesse en cas de tensions ou de conflits. Ces rapports de pouvoir impliquent que les entrevues faites avec des voyageurs sont teintées par la volonté de respecter le contrat d'accueil sousentendu par le réseau. Ainsi, aucune des personnes accueillies n'a refusé de faire une entrevue lorsque je leur ai demandé car, dans une logique de contre-don pour leur hébergeuse, il leur était difficile de dire non. De la même façon, il leur était presque impossible d'évoquer leur rapport à l'argent, presque tabou. Il était mal venu pour eux de le mentionner lors de l'entrevue, sous peine de passer pour des "profiteurs » qui n'ont pas compris l'« essence " du Couchsurfing, une image à laquelle les surfeurs ne souhaitent pas être associés face à leur hôtesse. Néanmoins, cette situation ne s'est pas révélée problématique s'agissant des questions sur leur visite de la ville, la sélection des profils, ainsi que leurs raisons « officielles» d'utiliser le réseau. De plus, les entretiens menés avec les hébergeurs étaient bien moins influencés par les rapports de pouvoir hospitaliers car les discussions avaient lieu chez eux. Cette méthodologie, imbriquée dans l'accueil, est très riche en vue de saisir les enjeux de l'hospitalité entre inconnus et, notamment, les représentations sociales que les membres ont de leur pratique.

\section{Une rencontre systématisée et sélectionnée entre inconnus biographiques}

6 Contrairement à ce que certains membres du réseau soutiennent, utiliser Couchsurfing ne garantit pas de rencontrer des individus différents de soi, mais constitue plutôt une forme d'entre-soi social. Si cela a déjà été montré par l'étude des réseaux en ligne (Cardon et al., 2014), cela demeure un élément primordial à comprendre et à décortiquer afin de saisir pourquoi et comment le réseau d'hospitalité fonctionne. Car s'il n'est pas nouveau que les touristes se réunissent souvent entre eux lorsqu'ils 
voyagent (Équipe MIT, 2002), il n'est pas fréquent que ces voyageurs s'accueillent chez eux et cohabitent durant leur séjour. Comprendre les similarités sociales qu'impliquent à la fois le réseau et la sélection des profils, c'est aussi comprendre une condition sine qua non de l'innovation touristique et hospitalière que représente Couchsurfing.

\section{"Strangers are just friends you haven't met yet! »}

7 Un grand nombre de communautés en ligne sont basées sur des relations qui ont majoritairement lieu hors-ligne, comme c'est le cas pour Facebook par exemple. Bien que Couchsurfing se situe dans la catégorie des sites qui, au contraire, font interagir des personnes qui ne se connaissaient pas auparavant, ce réseau s'inscrit dans une forme de continuité sociale avec ceux existant hors ligne. Ainsi, la sélection des interactions Couchsurfing, même si elle se fait en ligne, ne modifie pas certains déterminismes sociaux traditionnels, en particulier l'homophilie sociale, comprise ici comme le fait de fréquenter des personnes du même statut social que le sien (Bialski, 2012; Parodi, 2000). Le réseau d'hospitalité réunit donc des individus similaires socialement, jeunes, souvent issus des classes moyennes, parlant anglais et, dans le cas des surfeurs, aillant accès aux visas touristiques internationaux. Cette similarité est primordiale afin de comprendre comment l'hospitalité de Couchsurfing fonctionne: car sans cet entre-soi social, l'intérêt hospitalier de la rencontre entre couchsurfeurs diminuerait, voire disparaîtrait. En effet, les membres souhaitent rencontrer des individus qui les changent de leur réseau habituel, mais sans pour autant faire face à une altérité trop forte, sous peine d'être désagréable. Cette aspiration se retrouve dans les textes de présentation du réseau : les étrangers y sont décrits comme des «[...] friends you haven't met yet. $»^{4}$. La phrase a été rédigée dans une optique d'ouverture à la rencontre et de tolérance interactionnelle, centrale dans l'idéologie du réseau. Avec un regard sociologique, il est possible de la lire comme une description plutôt fine du genre de rencontres entre inconnus que le réseau crée. En effet, il est moins difficile de considérer quelqu'un qui nous est sociologiquement proche comme un potentiel ami et de cohabiter avec lui. Aussi, l'expression sous-entend que rencontrer un inconnu est accessible et que cela n'a juste pas encore été fait. C'est un autre avantage de l'hébergement Couchsurfing: l'échange entre les membres est systématisé et donc facilité par le passage en ligne. Le cadre d'interaction offert par le site permet de réduire le risque de ne pas trouver un logement pour les surfeurs et les conséquences sociales d'une trop grande différence entre les membres. En effet, les profils sont constitués de catégories orientant et soutenant la sélection entre internautes. Le premier onglet intitulé «About me » contient des descriptions multiples, dont, parmi les plus importantes : un autoportrait du couchsurfeur, les raisons pour lesquelles il utilise le réseau, ses films, musiques ou livres favoris, ou encore une expérience personnelle jugée extraordinaire par le membre. Vient ensuite l'onglet "Photo", où des images illustrant le profil sont disponibles; elles sont importantes car un profil sans photographies sera souvent considéré comme moins complet ou moins attractif. Puis, l'onglet "References », où les membres écrivent leur avis sur l'expérience vécue, est essentiel car il permet de hiérarchiser le profil en fonction de l'utilisation qui est attendue du réseau. Ce cadre technique assure une systématisation de la sélection entre couchsurfeurs. Cette systématisation offre une forme de sécurité interactionnelle qui participe à la faisabilité de l'hospitalité couchsurfing. De cette façon, le réseau permet d'éviter de devoir briser la glace avec des inconnus, comme l'explique une surfeuse « 
Couchsurfing is really a good 'socially acceptable excuse'»; plus besoin de prendre son courage à deux mains pour aller parler à un étranger, avec qui on ne partagera peutêtre que peu d'intérêts en commun, ou bien d'être incertain quant au lieu d'hébergement du soir, car le réseau atténue ces risques. Comme le décrit un hébergeur parisien, le poids de la recherche de l'autre n'est plus aussi fort qu'auparavant: " C'était... en rencontrant des gens en se mettant dans un café, en rencontrant des gens dans la rue, en discutant dans un musée ou quelque part, ça peut être l'employé de banque avec qui on discute parce qu'on fait la queue pendant deux heures pour récupérer des espèces [...], mais il n'y avait pas de site qui faisait un lien, le lien il fallait le chercher. ". Cette idée est très vraie pour les hébergeurs, mais parfois moins pour les surfeurs, particulièrement s'ils voyagent dans des grandes villes touristiques ${ }^{5}$. Ces derniers ont moins le choix, surtout si voyager avec Couchsurfing est une condition financière essentielle de leur voyage. Néanmoins, les touristes comme les résidents trouvent une cohérence dans l'hospitalité qui implique une rencontre moyennement risquée socialement, car basée sur des similarités sociales et automatisée par la sélection des rencontres en ligne.

\section{Une sélection par les profils}

8 Si certains ont avancé ailleurs l'importance des commentaires dans la constitution de Couchsurfing comme une communauté de personnes similaires (Germann Molz, 2013), notre recherche a mis l'emphase sur les profils et sur leur rôle dans la perpétuation de l'homophilie sociale du réseau. En effet, tout comme E. Illouz dans son étude à propos des sites de rencontre, nous pensons que l'utilisation de l'écrit pour se présenter en ligne promeut l'uniformité et la standardisation, car les membres tentent de produire le profil d'une personnalité « désirable» (Illouz, 2006, p. 249) en fonction des critères sociaux du site utilisé. Ces présentations de soi spécifiques aux réseaux en ligne permettent aux internautes de faire une sélection des personnes avec qui ils souhaitent interagir. Dans le cas de Couchsurfing, cette sélection se base sur des caractéristiques en ligne, plus spécifiquement, la réputation de la communauté et les compétences communicationnelles des membres (Kunz et al., 2015). Ainsi, tout comme lors d'une discussion en face à face les acteurs se fient à des indices communicationnels - comme, par exemple, un sourire -, l'échange en ligne requiert une maîtrise des indicateurs fournissant des informations sur la personne en face. Dans la description de soi écrite sur les profils, les couchsurfeurs vont beaucoup regarder les intérêts communs partagés. Ces descriptions recoupent des caractéristiques similaires d'un profil à l'autre; par exemple, une surfeuse allemande écrit: "My philosophy is to live and appreciate all the things it brings me, the most important to me is being positive, always enjoy what happens and meet new people from around the world!». On reconnaît là la description d'une personnalité « désirable » selon les critères des couchsurfeurs : ouverture à la rencontre, curiosité interculturelle et flexibilité. Toutefois des différences, notamment de goût, se manifestent dans les profils et vont permettre une sélection plus fine. Par exemple, un hébergeur parisien adorant la musique classique fait bien attention à sélectionner des surfeurs musiciens, voire idéalement des pianistes. Ainsi, en plus de la première sélection sociale qui s'opère par le simple fait de s'inscrire sur le site et de l'utiliser, une seconde sélection est mise en place au moment du choix du couchsurfeur. Les couchsurfeurs recherchent, encore une fois, une similarité, dans les goûts notamment, et non la rencontre avec une franche altérité. Comme les hébergeurs le disent bien, ils sont moins enclins à accueillir lorsqu'ils ne trouvent pas d'intérêts 
communs. "Alors que moi, déjà je choisis un peu les profils, voir par rapport aux affinités, parce que vraiment j'aime bien entretenir des contacts même par après avec des couchsurfeurs [...]. » De cette façon, les similarités de goûts sont un autre critère de sélection des profils. Si la recherche de similitudes orientait déjà les préférences sociales avant les réseaux en ligne, elle est dorénavant systématisée et automatisée, les goûts de chacun faisant partie d'une base de données visible, même si elle est mise en scène. La vraie différence concerne en fait la perception des membres, qui ont le sentiment de choisir de façon plus informée, plus élective les personnes qu'ils rencontrent. C'est pourquoi nous considérons les couchsurfeurs comme des "biographical stangers" selon la notion forgée par L. Lofland (1998), qui veut que des personnes soient inconnues lorsqu'elles ne partagent pas de passé personnel commun. Toutefois, les couchsurfeurs ne sont pas des "cultural strangers ", ne partageant aucun système de valeurs ou de références, bien au contraire. Le dispositif en ligne Couchsurfing favorise donc une mise en contact, incarnée dans une situation d'hospitalité temporaire, qui permet d'équilibrer la rencontre entre sa dimension étrangère et sa dimension familière.

\section{La situation d'hospitalité : lieu d'une résolution pratique entre l'altérité et son atténuation}

9 La tension structurante entre le familier et l'altérité est bien connue des études sur le tourisme puisque : «Par le seul fait de se déplacer temporairement vers des lieux du hors-quotidien, les touristes valorisent un différentiel d'altérité (la qualité de ce qui est autre) sans lequel ils n'auraient pas de raison de retrouver ailleurs ce dont ils pourraient se saisir ici. » (Coëffé, 2013, p. 12). Et de fait, cette tension est primordiale dans une recherche sur la rencontre entre touristes et résidents. Dans le cas de Couchsurfing, l'hospitalité se révèle source d'ambivalence, mais aussi d'équilibre entre quotidien et hors quotidien. De cette façon apparait un paradoxe : les couchsurfeurs semblent chercher un environnement qui leur est familier tout en désirant, dans le même temps, cette qualité de la différence primordiale au tourisme.

\section{Les ressources familières et sécurisantes : un lien social communautaire}

10 Ainsi, le contexte de cohabitation entre un résident et un touriste offre deux types de «ressources » familières aux membres: l'un basé sur la communauté Couchsurfing, l'autre sur l'assurance d'un lien social. Dans un premier temps, comme nous l'avons déjà évoqué, l'étranger que l'on rencontre possède des similarités sociologiques, qui atténuent l'écart social entre les membres et facilite le fait de vivre avec un inconnu. Mais, la fluidité des rencontres ne s'explique pas uniquement par cette homophilie sociale. Les couchsurfeurs développent, au fur et à mesure de leurs expériences, des compétences à la cohabitation et adoptent de plus en plus une culture Couchsurfing. Cette dernière est souvent dénommée par les membres le "Couchsurfing spirit", l'« esprit Couchsurfing " ou encore, comme le relève S. Buchberger (2013, p. 84) dans son étude des couchsurfeurs marocains, l'« idée du couch». Cette culture implique une certaine façon d'interagir avec les autres membres et un respect de règles, parfois implicites, qui souvent exigent des adaptations et des compromis pour les couchsurfeurs ne provenant pas de l'Occident ${ }^{6}$. Ainsi, les membres du réseau, bien 
qu'ils souhaitent interagir avec des inconnus, sélectionnent des personnes avec qui ils ont en commun, non pas la langue et les dieux comme c'était le cas dans l'hospitalité antique des Grecs (Boudou, 2013), mais le partage de l'adhésion au réseau d'hospitalité, ainsi que les valeurs et compétences que cette adhésion sous-entend. Ces similarités participent d'un processus d'atténuation de l'altérité de la rencontre entre inconnus et facilitent ainsi une relation hospitalière exigeante socialement. Le second type de ressources qu'implique le réseau concerne une forme d'atténuation de la solitude des surfeurs. L'une des données mises en lumière par le terrain concerne la relative précarité interactionnelle des voyageurs et les avantages que l'utilisation du réseau implique face à cette dernière. Les habitants en mouvement sont, en effet, fragiles puisqu'ils doivent comprendre des personnes qui leur sont différentes dans des lieux différents de ce qu'ils connaissent (Lazzarotti, 2014). Cette vulnérabilité est marquée chez des couchsurfeurs. Or l'hospitalité permet de relativiser cette précarité du touriste, notamment pour ceux des enquêtés dont le voyage s'étalait sur plusieurs mois. Comme l'une des surfeuses interrogées nous en a fait part: "I think if you are alone Couchsurfing is really great, like, it is fine to go eat a meal by yourself, go to a museum appreciate something, and have a nice moment by yourself, but sometimes it is nice to just be able to turn to someone next to you and be like "isn't this food great, isn't this painting amazing?" and just to share [...] ». Par ailleurs, vivre avec un résident parisien permet de pallier de nombreuses incertitudes logistiques concernant, par exemple, des connaissances pratiques à propos du métro, des horaires d'ouverture d'un musée, etc. D'autres interrogations sont plus culturelles: un enquêté d'origine sud-coréenne m'a un jour demandé pourquoi il y avait des vélos garés dans les rues de Paris que tout le monde pouvait utiliser, le concept du "Vélib' » lui étant inconnu. La relation avec l'hébergeur devient une manière d'être plus familier avec l'endroit visité, mais aussi une façon de ne pas être isolé dans une mobilité parfois solitaire. Cette analyse du lieu de refuge, qui temporise un certain isolement social et culturel, a été menée dans le contexte de la mobilité des étudiants à l'international (Pleyers et al., 2008). Toutefois, elle n'a jamais été étudiée en contexte d'hospitalité, comme c'est le cas dans Couchsurfing. Cela implique qu'outre la gratuité du réseau, l'aspiration au lien social, parfois difficile à susciter en situation de mobilité, revêt une importance forte dans la pratique. Cela étant dit, cette dimension rassurante de la rencontre doit être équilibrée par une dose de risque et de différence, sous peine de ne plus être touristique.

\section{Les ressources de l'altérité : le caractère irréductible de la singularité de l'hébergeur et de son lieu de vie.}

11 L'hospitalité, dont nous évoquions plus tôt les rapports de pouvoir, est une pratique particulièrement risquée et peut se retourner contre les personnes impliquées lorsqu'elles se connaissent avant de cohabiter (Gotman, 2001 ; Montandon, 2001). Cela est d'autant plus vrai lorsqu'il s'agit d'inconnus. C'est pourquoi la cohabitation Couchsurfing nécessite une forme de "sécurité interactionnelle", que l'homophilie sociale et communautaire soutient. Toutefois, il convient de souligner que si l'idéologie de la rencontre est si forte dans les discours des enquêtés, c'est aussi parce que les membres interagissent avec des personnes qu'ils ne connaissent pas. La sociologie, telle que pratiquée dans cette recherche, trouve ici une limite s'agissant de son analyse en termes de statuts sociaux et de similarités, car chaque individu rencontré demeure bel et bien un inconnu, que l'autre membre n'a jamais rencontré hors ligne. Cette prise en 
compte de l'originalité irréductible de chaque personne est essentielle afin de cerner ce qui importe aux couchsurfeurs : non pas le choc culturel et social de l'altérité humaine entière (comme prôné dans les discours), mais plutôt une altérité dont les angles sont arrondis et qui permet un changement de l'environnement interactionnel des membres. Ainsi, la personnalité de l'autre couchsurfeur représente une «dose» de différence, de hors-quotidien indispensable à l'expérience touristique. Cette aspiration prend la forme d'un discours sur la surprise, évoquée par les surfeurs et les hébergeurs lors des rencontres. Cette adrénaline, voire la légère anxiété ressentie avant la première interaction, est une dimension qui caractérise le voyage Couchsurfing, comme le souligne une surfeuse "I mean... you never really know who you are going to meet, so it is always a surprise to meet a couchsurfer, I really like travelling that way... it is challenging. » La surprise et le risque social qui l'accompagne sont souvent complètement acceptés, voire recherchés par les couchsurfeurs (Tan, 2013). Ceci explique pourquoi le thème de la confiance entre les membres n'est jamais fortement apparu dans les entretiens. Ainsi, dans les différences entre chaque hôte réside une aspiration à un changement d'univers relationnel, typique des couchsurfeurs. Cet alliage entre homophilie sociale et singularité de chaque individu, permis par Couchsurfing, permet de comprendre comment le familier et l'altérité sont modérés au cœur de la relation hospitalière. Si la rencontre est très présente dans les discours des surfeurs, la cohabitation chez un particulier joue aussi un rôle central dans leur quête de différence et dans la représentation qu'ils se font de leur voyage. De cette façon, le partage du lieu de vie de l'hébergeur est une source de nouveauté et de différence, par rapport à ce que les surfeurs connaissent. Beaucoup des touristes que j'ai accueillis tâtonnent dans cet espace qui leur est inconnu. Certains ne savent pas comment ouvrir la porte d'un immeuble parisien, dont le bouton d'ouverture peut être confondu avec un interrupteur pour la lumière; d'autres prennent des douches trop longues, vident le ballon d'eau chaude et finissent par se rincer à l'eau froide; ou encore, certains ont mal noté le code wifi et n'ont pas accès à Internet dans l'appartement. Toutes ces adaptations logistiques participent au différentiel d'altérité évoqué plus tôt et font que rencontrer et vivre chez un hébergeur devient une expérience touristique.

\section{Le Couchsurfing un tourisme de la rencontre}

12 Les couchsurfeurs, comme de nombreux visiteurs, sont pétris d'aspirations à la distinction touristique. Ils perçoivent leur pratique comme différente du tourisme dit de masse et surtout comme étant plus «alternative». En effet, le lieu de séjour offert par le réseau étant extérieur au marché professionnel de l'hospitalité, les membres considèrent, notamment, que l'originalité de leur pratique réside dans cet hébergement entre particuliers. Toutefois, selon eux, leur différenciation par rapport à la caricature $\mathrm{du}$ touriste visitant Paris en bus et photographiant de façon intempestive réside également grandement dans le fait de rencontrer un résident de la capitale.

\section{Le quotidien extraordinaire : personnalisation du séjour et « touristification » de la rencontre}

13 Ainsi, il existe dans le Couchsurfing une personnalisation de l'expérience touristique, non pas dans le sens où les visites sont plus adaptées aux aspirations et personnalités 
des visiteurs, mais car l'expérience touristique devient intimement liée à l'hébergeur et à sa rencontre. De cette façon, il est possible de ne pas apprécier une ville car l'interaction avec l'accueillant ne satisfait pas les critères interactionnels de Couchsurfing. Une surfeuse me décrit ainsi son expérience en demi-teinte en Allemagne : "In Berlin I had a host who was quiet, like very sweet, nice, but quiet. And there was conversation, but it was work, and yeah Berlin wad not my best city and I felt very lonely, like very isolated and lonely. I don't know, but... yeah, I think the host really, really has a huge effect on your perception of the city. » Cette personnalisation peut être poussée jusqu'à une forme de «touristification » de l'hébergeur et de son quotidien. J'ai de nombreuses fois été photographiée devant la tour Eiffel, ou encore devant la porte de mon appartement " pour le souvenir ». De la même façon, des actions relevant du quotidien, telles qu'aller en cours, prendre le petit déjeuner ou bien cuisiner, revêtent un sens touristique. Un surfeur taïwanais m'expliquait qu'il ne cuisinait jamais chez lui car, à Taiwan, la nourriture de rue revient moins chère que de préparer ses propres plats. Me voir cuisiner le repas du soir était pour lui une représentation du quotidien des Français. C'est pourquoi nous pensons que, dans le tourisme Couchsurfing, l'hébergeur lui-même revêt des caractéristiques touristiques, ce qui débouche sur une forme de tourisme de la rencontre. De cette façon, l'accueillant et sa vie quotidienne sont perçus comme un " réservoir » de la culture visitée et deviennent des propriétés touristiques. Le concept de «tourist gaze " permet de mieux éclairer ce phénomène. Selon J. Urry (1990), à l'origine de cette notion, c'est la distinction entre ce qui est ordinaire et extraordinaire qui constitue le regard touristique. Cette différence basique permet d'organiser, de modeler et de classifier le monde, mais n'est en aucun cas un simple regard sur une réalité qui existe déjà (Larsen, 2006). Dans le cas des surfeurs, le quotidien des hébergeurs devient extraordinaire puisqu'il est en dehors de leur univers familier. Leur «tourist gaze » met en forme la rencontre avec l'hébergeur et ses habitudes de vie afin de les catégoriser comme touristiques et «alternatives». Ce processus participe à une forme de "touristification» de la rencontre entre membres du réseau. Ainsi, l'interaction couchsurfing prend parfois le pas sur le lieu du séjour et l'échange avec l'hébergeur colore la visite au point où, par exemple, une banlieue belge a pu devenir attrayante pour cette voyageuse : "I stayed with another guy, and technically, it was the most boring night we were in the suburbs, we ate spaghetti for dinner, but it was so great ![...] I just liked his company and that changes the experience, I really liked the suburbs, which is usually the boring part of a city!». Les rencontres Couchsurfing débouchent ainsi sur l'imbrication du résident et de son lieu de vie dans la représentation touristique des surfeurs, leurs discours étant plus centrés sur les gens que sur les lieux, ce qui illustre le type de tourisme de la rencontre que crée le réseau d'hospitalité.

\section{Des visites parisiennes en décalage avec les représentations alternatives des membres}

On pourrait penser que ce tourisme personnalisé se prolonge dans la visite de Paris et que les couchsurfeurs, dans une optique de distinction, évitent les lieux comme la tour Eiffel et ses équivalents. Bien que certains surfeurs ne prennent pas de photographies durant leur séjour et que d'autres privilégient de longues marches dans la capitale où ils flânent plutôt que des voyages en métro d'une attraction à une autre, la grande majorité visite tout de même les lieux classiques du tourisme parisien. C'est ce qui est apparu de mes observations des surfeurs. Ces derniers ont souvent une idée des 
grandes visites qu'ils veulent faire avant d'arriver dans la ville et n'aspirent pas à des itinéraires touristiques en dehors des sentiers battus. Il est également intéressant de constater que les hôtes parisiens, qui adhèrent pourtant au discours de distinction du réseau, suivent ce schéma de visites et ne modifient en rien les envies des surfeurs : " Sinon, on a eu deux Américaines avec qui c'était super, vraiment elles étaient dans l'esprit Couchsurfing [...]. Elles voulaient visiter quelques trucs, elles voulaient aller à la tour Eiffel, alors on les a emmenées à la tour Eiffel, on a fait les sorties phares qu'elles voulaient, [...], elles étaient ravies. » Bien sûr, puisque le maître de maison et l'invité passent du temps ensemble, le résident va souvent faire découvrir des lieux faisant partie de son expérience personnelle de la ville, tel que le café ou l'épicerie du quartier, et l'endroit revêt donc un caractère «local » pour les voyageurs (Bialski, 2009; Zuev, 2012). Mais les activités touristiques des membres, si elles incluent des représentations de l'interaction avec l'hébergeur, ne sont pas majoritairement constituées de ces lieux. Les photographies collectées auprès des voyageurs après leur séjour l'illustrent bien: on y trouve notamment des clichés de la tour Eiffel, de la cathédrale Notre-Dame ou de Versailles. Les hébergeurs eux-mêmes jouent le jeu des attractions "à voir", ce qui soutient l'hypothèse selon laquelle les hébergeurs "routinisent " leurs modes d'accueil (Zuev, 2013). Ceux qui précédemment défendaient le caractère unique des expériences Couchsurfing en tant que surfeurs décrivent quelques minutes plus tard leurs routines hospitalières et touristiques. Cela vaut pour Paris comme pour d'autres villes d'Europe de l'Ouest. Un surfeur belge m'explique par exemple la façon dont il fait généralement découvrir sa ville à ses invités : «Donc Bruxelles n'est pas énorme, donc c'est le tour typique, c'est une promenade qui va de X ̀̀ Y. C'est un tour qui commence à la Grand-place et puis qui va vers Manneken-Pis, le petit bonhomme qui fait pipi, et puis quelques autres points clés qui terminent alors dans un bar qui s'appelle: le bar Délirium où il a 2000 sortes de bières [...]. » Comment se fait-il que cet écart entre les discours et les activités touristiques n'amoindrisse en rien la représentation alternative que les couchsurfeurs ont de leur pratique? Il s'avère que, plus qu'un tourisme urbain, le Couchsurfing permet un tourisme de la rencontre entre résident et voyageurs. Une rencontre entre personnes similaires socialement, dans un contexte de cohabitation temporaire, mais une rencontre entre inconnus tout de même. Certaines recherches affirment que les couchsurfeurs s'éloignent du séjour traditionnellement centré sur des destinations à visiter et se tournent vers un tourisme plus «intimiste", dans lequel l'interaction émotionnelle entre membres est essentielle et déboucherait sur un «tourisme de sensations » (Bialski, 2009). Si cette thèse peut se révéler pertinente pour décrire certaines rencontres entre couchsurfeurs, nous pensons qu'elle n'englobe pas suffisamment la variété de leurs échanges, particulièrement lorsque les expériences de cohabitation sont négatives ou encore lorsque les supposées relations émotionnelles n'ont pas lieu. Nous croyons plutôt que la focale touristique se déplace, pas forcément vers une découverte en termes d'émotions mais vers la visite de quelqu'un et de son existence là où, auparavant, le but du voyage était la découverte de lieux et d'attractions. Une surfeuse étatsunienne m'explique par exemple: «[...] And I think, yeah, when you are traveling especially for a long time, it's really nice to be in a home, and feel really comfortable and just blend into someone's life almost.» Cela va donc au-delà de la rencontre avec un inconnu biographique. Il s'agit rendre visite à quelqu'un et de s'immerger temporairement dans son existence ${ }^{7}$. Cette immersion est en rapport avec la distinction que les couchsurfeurs font entre eux et les "autres touristes ». Ces derniers n'auraient accès qu'aux lieux et n'auraient de liens sociaux qu'ancrés dans 
l'industrie touristique, avec des guides par exemple, ou encore avec d'autres touristes dans une auberge de jeunesse. Comme le décrit un surfeur: "I think in a hostel the conversation is very specific and surfacy. You are going to a hostel for a specific purpose, you want to meet other people but they won't be your friends [...]. Whereas with Couchsurfing [...] you have already made clear that, like: I think you are very interesting, I want to come stay with you, I think we could be friends. " Les couchsurfeurs auraient, quant à eux, accès à des relations " authentiques », hors des échanges touristiques marchands. Ainsi, c'est dans ce basculement d'un tourisme des lieux à un tourisme de l'existence quotidienne que réside toute l'innovation touristique du réseau, et non, concrètement, dans le type de visites touristiques des membres.

\section{Conclusion}

$\mathrm{Si}$, au premier abord, on peut penser que l'innovation de Couchsurfing réside dans la mise en contact entre des inconnus via Internet, en réalité c'est surtout dans la systématisation de l'hospitalité entre inconnus et dans l'imbrication entre cette rencontre hospitalière et des projets touristiques qu'elle se situe. La formule proposée par le réseau permet une cohabitation entre des personnes qui ne se connaissent pas, mais dont la proximité sociologique atténue les risques inhérents à l'hospitalité. Cette base de communauté en ligne permet une expérience touristique source d'altérité maîtrisée. Ainsi, les rencontres entre touristes et résidents autorisent les surfeurs à percevoir leur pratique comme alternative, puisque personnalisée, tout en gardant des consommations culturelles parisiennes "classiques». Cette pirouette intellectuelle n'est possible que grâce à la transversalité de la rencontre entre les membres, qui permet de constituer un espace d'équilibre et de tension entre le familier et l'altérité, espace qui n'était pas aussi accessible avant l'invention des réseaux d'hospitalité en ligne.

\section{BIBLIOGRAPHIE}

Paula BIALSKI, Intimate tourism: Enquête dans un réseau d'hospitalité, Éditions Solilang, 2009.

Paula BIALSKI, Becoming Intimately Mobile, Peter Lang, 2012.

Benjamin Boudou, « Ennemis, hôtes et étrangers. Enquête sur les identités politiques grecque et romaine », Mots. Les langages du politique, n 101, 2013.

Sonja BUCHBERGER, « Rooted Cosmopolitanisms, Deceived Kinship and Uneasy Hospitality among Couchsurfers in Tunisia ", dans David PICARD, Sonja BUCHBERGER (dir.), Couchsurfing

Cosmopolitanisms, Can Tourism Make a Better World?, Transcript, 2013.

Dominique CARDON et Zbigniew SMOREDA, « Réseaux et les mutations de la sociabilité », Réseaux, $n^{\circ} 2,2014$. 
De-Jung CHEN, « Global concept, local practice: Taiwanese experience of CouchSurfing », Hospitality \& Society, $\mathrm{n}^{\circ} 3,2012$.

Vincent COËFFÉ, « Lieux touristiques et gestion de l'altérité. Réflexions autour des pratiques de sociabilité », Mondes du Tourisme, n 8, 2013.

Anne DURFouRMANTELLE et Jacques DERRIDA, De l'hospitalité, Calmann-Lévy, 1997.

ÉQUIPE MIT, Tourismes 1. Lieux communs, Belin, 2002.

Jeanne FAVRET-SAADA, « Être affecté », Gradhiva. Revue d'histoire et d'archives de l'anthropologie, 1990.

Jennie GERMANN MOLZ, "Cosmopolitans on the Couch:Mobile Hospitality and the Internet ", dans David PICARD, Sonja BUCHBERGER (dir.), Couchsurfing Cosmopolitanisms, Can Tourism Make a Better World?, Transcript, 2013.

Jacques T. GodBout, « Recevoir, c'est donner », Communications, n 65, 1997.

Anne Gotman, Le sens de l'hospitalité. Essai sur les fondements sociaux de l'accueil de l'autre, Presses universitaires de France, 2001

Marie-Claire GRASSI, « Pour une histoire de l'hospitalité », dans Alain MONTANDON (dir.), Lieux d'hospitalité : hospices, hôpital, hostellerie, Presses universitaires Blaise Pascal, 2001.

Eva ILLOUZ, « Réseaux amoureux sur Internet », Réseaux, n 4, 2006.

Werner KUNZ et Sukanya SESHADRI, «From virtual travelers to real friends: Relationship-building insights from an online travel community », Journal of Business Research, n 9, 2015.

Olivier LAZZAROTTI, « Un monde d'habitants mobiles », Dailymotion, 2014.

Lyn H. LOFLAND, The public realm: Exploring the city's quintessential social territory, Transaction Publishers, 1998.

Alain MONTANDON, «Lieux d'hospitalité », dans Alain MONTANDON (dir.), Lieux d'hospitalité : hospices, hôpital, hostellerie, Presses Universitaires Blaise Pascal, 2001.

Pierre PAILlÉ et Alex MUCCHIELLI, L'analyse qualitative en sciences humaines et sociales, Armand Colin, 2008.

Maxime PARODI, « La lente évolution de la sociabilité », Revue de l'OFCE, $\mathrm{n}^{\circ}$ 1, 2000

Geoffrey PLEYERS et Jean-François GUILLAUME, « Expériences de mobilité étudiante et construction de soi », Agora débats/jeunesses, nº 4, 2008.

Bernard SCHÉOU, « Couchsurfing et les réseaux de partage réinventent l'hospitalité », Espaces, $n^{\circ} 316,2014$.

Bastien SOULET et Cédric RICHET, « Moniteur sportif et sociologue : Récit d'une observation participante clandestine », Bulletin de Méthodologie Sociologique, n 1, 2010.

Jun-E TAN, « Cosmopolitanism as Subcultural Capital: Trust, Performance and Taboo at Couchsurfing.org », dans David PICARD et Sonja BUCHBERGER (dir.), Couchsurfing Cosmopolitanisms, Can Tourism Make a Better World?, Transcript, 2013.

Jean-Didier URBAIN, L'idiot du voyage. Histoires de touristes, Plon, 1991.

John URRY, The Tourist Gaze: Leisure and Travel in Contemporary Societies, London : Sage, 1990.

Dennis ZUEV, « CouchSurfing as a spatial practice: Accessing and producing xenotopos », Hospitality \& Society, $\mathrm{n}^{\circ} 3,2012$. 
Dennis ZUEV, « Hosting Marco in Siberia: Couchsurfing Hospitality in an 'Out of the Way' Place », dans David PICARD et Sonja BUCHBERGER (dir.), Couchsurfing Cosmopolitanisms. Can Tourism Make a Better World?, Transcript, 2013.

\section{NOTES}

1. On parle souvent de canapés pour évoquer l'endroit où l'invité dort, cet emplacement peut cependant prendre de nombreuses autres formes (lit, matelas sur le sol, chambre personnelle, etc.).

2. À noter que, tout comme les statistiques évoquées plus tôt, ces chiffres ont été produits par Couchsurfing à partir de leur base de données, leur constitution est donc obscure. Toutefois, ces résultats recoupent très bien la réalité que nous avons pu observer.

3. La notion d'équilibre, telle qu'elle est pensée ici, ne renvoie pas à un état de repos stabilisé et obtenu par l'égalité de deux poids, mais plutôt à un jeu de forces où tensions et déséquilibres ont bien lieu. Il s'agit ici plutôt d'insister sur la dimension « viable » de la pratique de Couchsurfing qui équilibre, de manière suffisante, l'altérité et la similarité pour perdurer.

4. L'expression vient de l'écrivain Wiliam Butler Yeast: "There are no strangers here; Only friends you haven't met yet. » Couchsurfing a réemployé une partie de cette citation sur sa page «About Us » : http://www.couchsurfing.com/about/about-us/.

5. Londres et Paris, par exemple, ont la réputation d'être des villes dans lesquelles il est très difficile de trouver un hébergeur, car le ratio d'hôtes et de surfeurs est inégal.

6. Pour approfondir cette idée d'une culture Couchsurfing à connotation occidentale voir notamment : Chen, 2012 ; Buchberger, 2013.

7. À noter que de 2013 à 2014, le slogan affiché sur la page d'accueil du réseau était «Share your life ». Aujourd'hui, il a été remplacé par : «Stay with Locals and Meet Travelers. Share Authentic Travel Experiences ». Voir : http://www.couchsurfing.com/about/about-us/

\section{RÉSUMÉS}

Grâce à l'avènement du Web 2.0, nombre d'internautes ont pu changer leur rapport à la mobilité, notamment leurs manières de voyager. L'émergence de réseaux d'hospitalité, dont le plus connu est Couchsurfing, atteste des réinterprétations du tourisme que permet Internet. Ce réseau, créé en 2004, met en relation des voyageurs avec des hébergeurs d'accord pour les accueillir gratuitement pour quelques jours. Le fonctionnement en ligne ainsi que la gratuité du réseau en font un service populaire, dont le nombre d'inscrits ne cesse d'augmenter. Cette formule de voyage est plébiscitée par ses membres, notamment grâce à l'expérience "alternative » et personnalisée que promeut le réseau. Si les couchsurfeurs sont convaincus du caractère unique et original de leur pratique, nous pouvons nous interroger sur le type d'innovations et de modifications impliquées par le réseau d'hospitalité. En nous basant sur une étude auprès de couchsurfeurs parisiens, nous mettons en lumière la manière dont les rencontres entre les membres modèlent l'expérience touristique des voyageurs. Le présent travail s'attache ainsi à dresser des pistes de compréhension des rencontres touristiques suscitées par les réseaux d'hospitalité en ligne et particulièrement de leur rapport à la familiarité et l'altérité. 
With the advent of Web 2.0, a substantial number of internet users changed their relationship to mobility, including the way they travel. The emergence of hospitality networks, and most notably Couchsurfing, demonstrates how the internet has allowed for reinterpretations of tourism. Created in 2004, this network connects travelers with hosts willing to accommodate them free of charge for a few days. The cost-free and online nature of Couchsurfing has contributed to its growing popularity, leading to a steadily increasing number of users. This way of traveling is particularly acclaimed by members because of the "alternative" and personalized experience promoted by the network. If couchsurfers are convinced of the uniqueness and originality of their practice, we may question the type of innovations and modifications implied by hospitality networks. Based on a study among Parisian couchsurfers, we illustrate how encounters between members shape the touristic experience of travelers. Thus, this article focuses on new ways of understanding the touristic encounters generated by online hospitality networks, and especially their relationship to familiarity and alterity.

\section{AUTEUR}

\section{PAULINE NEVEU}

Doctorante au département de sociologie de l'Université du Québec à Montréal

Courriel : neveu.pauline_marie@courrier.uqam.ca 